\title{
Minimizing the uncertainty about the uncertainty principle
}

\section{G. Vemulapalli, A. Marathay}

G. K. Vemulapalli, A. S. Marathay, "Minimizing the uncertainty about the uncertainty principle," Proc. SPIE 9663, Eighth International Topical Meeting on Education and Training in Optics and Photonics, 96630A (6 October 2003); doi: $10.1117 / 12.2208375$

SPIE Event: Eighth International Topical Meeting on Education and Training in Optics and Photonics, 2003, Tucson, Arizona, United States 


\title{
Minimizing the uncertainty about the uncertainty principle
}

\author{
G. K. Vemulapalli \\ Department of Chemistry, University of Arizona, 1306 E. University Blvd, Tucson, AZ 85721 \\ telephone: 520-621-6350; fax: 520-621-8407; e-mail: gkv@u.arizona.edu
}

\author{
A. S. Marathay \\ Optical Sciences Center, University of Arizona, Tucson, AZ 85721 \\ e-mail: marathay@u.arizona.edu
}

\begin{abstract}
Uncertainty principle forms a crucial part of quantum theory and wave optics. By using the propagation of a scalar, 3-D wave packet with and without dispersion as a heuristic device, we examine different aspects of the principle as it applies to transverse and longitudinal dispersion, and to time-dependent and stationary states.

(C)2003 Optical Society of America

OCIS codes: (000.2060) Education; (350.7420) Waves
\end{abstract}

\section{Summary}

A firm understanding of the uncertainty principle, both on qualitative and quantitative level, is essential for mastery of quantum theory, wave and quantum optics. Nevertheless many students are confused about what the principle actually states, very likely because of its historical developments [1,2]. Heisenberg's original formulation [3], with $\gamma$-ray microscope as an example, considered the distribution of positions and momenta in an ensemble of particles following a scattering experiment. Later Heisenberg [4] and Kennard [5] demonstrated that uncertainty principle results from a Fourier transform relation between momentum and position distribution functions.

When these two aspects of the uncertainty principle are lumped together, as it is done often, needless confusion arises. One-dimensional wave packets used as models in textbooks [6] are inadequate to illustrate the full scope of the principle.

We derive the appropriate amplitude function to describe the propagation in space and time of a 3-D wave packet that exhibits both the longitudinal and transverse dispersion. With this amplitude function and the corresponding probability density function, one can give a clear account of the uncertainty principle and how it leads to different interpretations depending upon whether one is describing an individual quantum state or an ensemble of particles.

\section{References}

1. Raymer, M. G. "Uncertainty Principle for Joint Measurement of Noncommuting Variables," Am. J. Phys. 62 (11), 986-993 (1994).

2. Hilgevoord, J and Uffink, J. “A New View of the Uncertainty Principle,” in Sixty-two years of uncertainty, A. I. Miller, ed., Plenum Press (New York, 1990), 121-137.

3. Heisenberg, W. "Uber den anschaulichen Inhalt der Quantentheoretischen Kinematik und Mechanik," Z. Phys. 43, 172-198 (1927).

4. Heisenberg W. The Physical Principles of Quantum Theory (Dover, New York, 1930).

5. Kennard, E. H. “Zur Quantenmechanik einfacher Bewegungstypen,” Z. Phys. 44, 326-352 (1927).

6. Cohen-Tannoudji, C, Diu, B and Laloë, F, Quantum Mechanics (Wiley, New York, 1977). 